\title{
Big Five model and trait emotional intelligence in
}

\section{camouflaging behaviours in autism}

\author{
Erin Robinson ${ }^{1}$, Laura Hull ${ }^{\text {la }}$, \& K. V. Petrides ${ }^{2}$ \\ 'Department of Psychology, University College London \\ 2London Psychometric Laboratory, University College London
}

a Corresponding author:

laura.hull.14@ucl.ac.uk

Department of Psychology, University College London

26 Bedford Way

London WC1H 0AP

+442076795365 


\title{
$\underline{\text { Big Five model and trait emotional intelligence in camouflaging }}$ behaviours in autism
}

\begin{abstract}
This study investigated the role of the five-factor model and trait emotional intelligence (trait EI; Petrides, Pita, and Kokkinaki, 2007) in camouflaging behaviours in a large sample of 278 autistic and 230 typically developing (TD) participants. Participants completed questionnaires assessing autistic traits, five-factor personality, trait EI, and social camouflaging of autistic traits. Associations between the personality dimensions of extraversion, agreeableness, conscientiousness, and neuroticism with autistic traits were found, supporting previous research showing that individuals with higher autistic traits are predisposed to specific personality traits. Trait EI had a negative relationship with autistic traits, suggesting a specific emotional difficulty for individuals with high autistic traits. Camouflaging was negatively related to extraversion, agreeableness, and conscientiousness, while it was positively related to neuroticism. Trait EI was also negatively linked to camouflaging. The differences between autistic and TD participants regarding camouflaging are discussed.
\end{abstract}

Keywords: autism, personality, trait emotion intelligence, camouflaging 

Autism Spectrum Disorders (ASD) are a group of developmental disorders diagnosed by an individual having persistent deficits across social settings (e.g. in communication and interaction) and showing a restricted, repetitive pattern of behaviour, interests, or activities (American Psychiatric Association, 2013). In addition to clinical diagnoses of ASD, autistic traits (such as difficulties with communication, strict behavioural routines, and aversion to change) are distributed across the entire population (Rotatori \& Deisinger, 2015). Throughout this paper we refer to people diagnosed with ASD as autistic individuals, following research suggesting that the majority of the autism community prefer an identity first description (Kenny et al., 2016).

\section{$\underline{\text { Five-Factor Model and Autistic Traits }}$}

The five-factor model of personality (consisting of the factors extraversion, neuroticism, agreeableness, conscientiousness, and openness to experience) is commonly used to ascertain an individual's personality type. A recent meta-analysis has synthesised research on autism and five-factor personality (Lodi-Smith, Rodgers, Cunningham, Lopata, \& Thomeer, 2018). In autistic individuals, all five factors of personality were negatively associated with autistic characteristics (note, they used the reverse of neuroticism - emotional stability). Autistic individuals were also found to have significantly lower scores on all five factors than typically developing (TD) individuals. The largest of these effects were for extraversion, emotional stability (neuroticism), and agreeableness.

\section{$\underline{\text { Trait EI and Autistic Traits }}$}

Trait EI is defined as a constellation of emotional perceptions assessed via questionnaires and rating scales (Petrides, Pita, and Kokkinaki, 2007). It comprises all of the personality facets that relate to affect. Trait EI is of interest because there is clear evidence of emotional differences in autistic individuals, such as difficulties with empathy (Baron-Cohen \& 
Wheelwright, 2004), and facial or vocal emotion identification (Golan, Baron-Cohen \& Hill, 2006). Autistic individuals have demonstrated lower trait EI than TD controls (Petrides et al, 2011; Boily, Kingston, \& Montgomery, 2017). Gökçen, Petrides, Hudry, Frederickson, and Smillie (2014) reported a negative correlation between autistic traits and trait EI, as well as the wellbeing, sociability, and emotionality factors of trait EI. However, Gökçen et al. (2014) used TD participants and, therefore, the association with autistic traits has not yet been established in an autistic sample.

\section{Personality, Trait EI and Camouflaging}

Camouflaging, defined as the use of compensation and masking strategies in social situations, is a relatively new area of research in ASD. It includes hiding autistic characteristics, using techniques to appear socially competent, and preventing others from seeing social difficulties (Hull et al., 2017). Camouflaging is conceptually related to reputation management in TD individuals (Izuma, Matsumoto, Camerer, \& Adolphs, 2011). Reputation management (also known as self-presentation) involves various strategies to both motivate and construct one's public reputation, which may be distinct to one's private self-impression (Leary \& Kowalski, 1990).

Camouflaging is not specific to autistic individuals. The continuous nature of autistic traits within the population means that TD individuals also camouflage these traits. A recent study found comparable underlying dimensions of camouflaging in TD individuals and autistic individuals (Hull et al., 2018), although the latter camouflage at significantly greater levels. As camouflaging is still a relatively new area of research, there has not yet been any investigation of the role of personality. Personality has, however, been studied in relation to self-presentation strategies in TD samples which, as previously mentioned, may overlap conceptually with camouflaging. 
Extraversion has several facets that are prima facie relevant to self-presentation strategies. For example, extraverted individuals make friends easily and enjoy being around large groups, contexts in which self-presentation strategies may be employed. Early research found that extraversion was positively associated with public self-consciousness (Trapnell \& Campbell, 1999) and with self-monitoring (John, Cheek, \& Klohnen, 1996), both of which are related to self-presentation strategies. Extraversion has also been linked to actual selfpresentation and emotional disclosure (Seidman, 2013).

Neuroticism is positively associated with online self-presentation behaviours (Seidman, 2013). The relationship between other personality traits and self-presentation is still uncertain. Agreeableness has been positively associated with actual self-presentation but negatively with attention-seeking, while conscientiousness was negatively associated with several self-presentation behaviours, such as attention-seeking and ideal self-expression (Seidman, 2013). The same study found no relationship between openness to experience and self-presentation.

A link may also exist between trait EI and camouflaging, based on studies examining the role of trait EI in TD self-presentation. High trait EI individuals score higher on measures of self-monitoring (Schutte et al., 2001), while Petrides, Pérez-González, and Furnham (2007) found trait EI to be a positive predictor of the ability to modify self-presentation. However, both these studies examined self-presentation as part of the larger concept of selfmonitoring. This concept also encompasses sensitivity to emotional expression, which is shown to be impaired in autistic individuals (Baron-Cohen \& Wheelwright, 2004; Golan et al., 2006). Research has demonstrated greater camouflaging in autistic individuals (Hull et al., 2018), therefore the relationships between personality, trait EI, and self-presentation in TD populations may not extend to camouflaging by autistic individuals. 


\section{The Current Study}

The current study is the first to explore the relationship between the five-factor model of personality, trait EI, and camouflaging in autistic and non-autistic individuals. It attempts to elucidate the roles of trait EI and personality in camouflaging. It also advances research on trait EI and autism by investigating the predictive role of trait EI in autistic traits using large autistic and non-autistic samples for the first time. The study used a correlational design to assess the associations between the five-factor model, trait EI, autistic traits, and camouflaging.

The following hypotheses were tested:

Individuals with higher autistic traits will be:

H1: Higher on neuroticism; lower on extraversion, agreeableness, and conscientiousness

\section{H2: Lower on trait EI}

Individuals with greater camouflaging will be:

H3: Higher on extraversion

H4: Higher on neuroticism

H5: Lower on conscientiousness

In addition, exploratory analyses were performed on the relationships between autistic traits, personality, trait EI, and camouflaging. 


\section{$\underline{\text { Participants }}$}

A power analysis was conducted prior to data collection. This indicated that at least 300 participants were needed to detect effect sizes similar to previous research. A large sample of responses from 592 participants were collected (404 females, 172 males, mean age=36.8 years, $\mathrm{SD}=15.4$ ). Of them, 278 reported having a diagnosis of an ASD (further reporting the age at which they were diagnosed and the type of healthcare professional who diagnosed them), 230 were TD, and 84 considered themselves to have self-diagnosed ASD. Selfdiagnosed participants were not included in any analyses. Participants were recruited through an online recruitment system at [institution removed for review], via social media, and from the [database removed for review]. The participants recruited through [institution] $(\mathrm{N}=58)$ were rewarded with course credit. Most participants were from North America, Europe, or Australia; however, there were also participants from Asia (N=37), South America $(\mathrm{N}=3)$, and South Africa $(\mathrm{N}=2)$.

\section{$\underline{\text { Measures }}$}

The experiment employed four questionnaires, presented to the participants in an online survey through Qualtrics.

\section{The Broad Autism Phenotypes Questionnaire (BAPQ; Hurley et al., 2007)}

The BAPQ was used to measure participants' autistic traits. Internal reliability was high $(\mathrm{a}=.96)$. The questionnaire consists of 36 closed questions scored on a 6-point scale and contains three subscales: aloofness ( 12 items, $a=.93$ ), rigidity ( 12 items, $a=.92$ ), and pragmatic language (12 items, a $=.90$ ). Scores above a certain cut-off (male average $=3.47$, female average $=3.19$ ) are associated with the Broader Autism Phenotype in the general population (Sasson et al., 2013). 
The Big Five Inventory (BFI; John and Srivastava, 1999)

The BFI was used as a measure of the five-factor model. The questionnaire consists of 44 closed questions scored on a 5-point scale. This measure was chosen because it is concise, thus reducing the likelihood of respondent fatigue, and shows high convergence with the NEO PI-R facets (Soto \& John, 2009). Cronbach's alphas for extraversion, neuroticism, conscientiousness, agreeableness, and openness to experience were $0.85,0.87,0.82,0.79$, and 0.81 , respectively.

Trait Emotional Intelligence Questionnaire (TEIQue-SF; Petrides, 2009).

The short form version of the TEIQue, consisting of 30 closed questions scored on a 7-point scale, was used to minimise respondent time. Internal consistency was high for the global score $(\mathrm{a}=.92)$ as well as for its four factors: emotionality ( 8 items, $\mathrm{a}=.82)$, sociability ( 6 items, $a=.79$ ), well-being (6 items, $a=.88)$, and self-control (6 items, $a=.75)$.

\section{Camouflaging of Autistic Traits Questionnaire (CAT-Q; Hull et al., 2018)}

The CAT-Q is a self-report measure of camouflaging, which consists of 25 closed questions scored on a 7-point scale. Internal consistency was high $(\mathrm{a}=.94)$ and there are three subscales: compensation ( 9 items, $a=.94)$, masking ( 8 items, $a=.80$ ), and assimilation ( 8 items, $\mathrm{a}=.90)$.

\section{$\underline{\text { Procedure }}$}

Participants completed the BAPQ first, followed by the BFI, then CAT-Q, and finally the TEIQue-SF. They were then asked for demographic details, including age, gender, nationality, native language, student status, education level, and type of ASD diagnosis (e.g. Autism, Aspergers, etc.). Finally, participants were debriefed on the complete aims of the study and were provided with the details of researchers. 
T-tests were undertaken to determine whether autistic and TD groups were significantly different on BAPQ and TEIQue-SF scores. Four multiple regressions were undertaken. The first two regressed BAPQ scores on the four factors of trait EI separately in the autistic and TD samples. The third and fourth regressed camouflaging on BAPQ subscale scores, BFI variables, and the trait EI factors, separately in the autistic and TD samples. In these latter two regressions, sets of variables were entered in decreasing order of the strength of correlation with the CAT-Q total score. 


\section{Results}

Table 1

Means and SD for the BAPQ, BFI, TEIQue-SF, and standardised CAT-Q

\begin{tabular}{|c|c|c|c|}
\hline Measure & $\begin{array}{c}\text { All } \\
\text { Participants } \\
\text { (SD) }\end{array}$ & $\begin{array}{c}\text { Typically } \\
\text { Developing } \\
\text { (SD) }\end{array}$ & $\begin{array}{c}\text { Autistic } \\
\text { (SD) }\end{array}$ \\
\hline BAPQ & $3.86(0.92)$ & $3.12(0.79)$ & $4.31(0.68)$ \\
\hline Aloof & $4.05(1.06)$ & $3.32(1.00)$ & $4.48(0.85)$ \\
\hline Pragmatic Lang & $3.58(1.00)$ & $2.81(0.84)$ & $4.05(0.76)$ \\
\hline Rigid & $3.95(1.02)$ & $3.24(.90)$ & $4.40(0.86)$ \\
\hline \multicolumn{4}{|l|}{ BFI } \\
\hline $\mathrm{E}(1.00-5.00)$ & $2.43(0.89)$ & $2.81(0.92)$ & $2.21(0.79)$ \\
\hline $\mathrm{A}(1.11-5.00)$ & $3.46(0.75)$ & $3.68(0.70)$ & $3.33(0.78)$ \\
\hline $\mathrm{C}(1.00-5.00)$ & $3.47(0.79)$ & $3.58(0.77)$ & $3.46(0.78)$ \\
\hline $\mathrm{N}(1.00-5.00)$ & $3.65(0.90)$ & $3.33(0.92)$ & $3.87(0.81)$ \\
\hline $\mathrm{O}(1.30-5.00)$ & $3.67(0.72)$ & $3.67(0.70)$ & $3.64(0.75)$ \\
\hline TEIQue-SF & $3.96(1.02)$ & $4.60(0.94)$ & $3.54(0.86)$ \\
\hline Emotionality & $3.89(1.31)$ & $4.78(1.17)$ & $3.36(1.10)$ \\
\hline Sociability & $3.85(1.25)$ & $4.42(1.15)$ & $3.47(1.18)$ \\
\hline Well-being & $4.46(1.39)$ & $4.99(1.25)$ & $4.08(1.41)$ \\
\hline Self-Control & $3.63(1.17)$ & $4.09(1.13)$ & $3.32(1.11)$ \\
\hline CAT-Q & $4.39(1.24)$ & $3.64(1.14)$ & $4.84(1.05)$ \\
\hline Compensation & $4.03(1.64)$ & $2.97(1.41)$ & $4.67(1.41)$ \\
\hline Masking & $4.38(1.25)$ & $4.23(1.15)$ & $4.46(1.31)$ \\
\hline Assimilation & $4.86(1.48)$ & $3.89(1.47)$ & $5.44(1.16)$ \\
\hline
\end{tabular}

$B A P Q=$ Broad Autism Phenotype Questionnaire (Range 1-6); BFI = Big Five Inventory (Range 1-5); TEIQue-SF = Trait Emotional Intelligence Questionnaire -Short Form (Range 1-7); standardised CAT-Q = Camouflaging Autistic Traits Questionnaire (Range 1-7) 
Descriptive statistics for the BAPQ, BFI, TEIQue-SF, and CAT-Q are displayed in Table 1. Inspection of histograms showed the results for all four questionnaires to be normally distributed. Bonferroni corrections were used on all analyses. Correlations were assessed against $p=0.000175$, ANOVAs against $p=0.0025$, and t-tests against $p=0.00227$.

A t-test on BAPQ scores $[t(506)=18.19 ; p<.001 ; d=1.61]$ found autistic participants had significantly higher levels of autistic traits than TD participants. For autistic individuals $12.5 \%$ of male participants and $1.84 \%$ of female participants were below the cut-off score for BAP. For TD individuals, $60 \%$ of male participants and $54.01 \%$ of female participants were below the cut-off.

Another t-test was run to determine group differences in trait EI. This was found to be significant $[t(506)=13.26 ; p<.001 ; d=1.18]$, with TD participants scoring higher than autistic participants. This pattern of results held throughout the four trait EI factors.

\section{$\underline{\text { Five-Factor Model and Autistic Traits }}$}

Correlations between all variables and subscales are reported in Supplementary Table 2. Correlations between BAPQ scores and the personality domains of extraversion, agreeableness, and neuroticism were all significant in both samples. In support of Hypothesis 1, neuroticism was positively associated with autistic traits in both samples, while extraversion and agreeableness were negatively associated with autistic traits in both samples, thus supporting Hypotheses 2 and 3. Hypothesis 4 was not supported, as no relationship between autistic traits and conscientiousness emerged in either sample.

\section{$\underline{\text { Trait Emotional Intelligence and Autistic Traits }}$}

A negative correlation between scores on the BAPQ and TEIQue-SF was found in both the autistic and TD samples, supporting Hypothesis 5. Negative correlations were also found between BAPQ scores and all four factors of TEIQue across autistic and TD participants. 
Multiple regressions were undertaken of BAPQ scores on the four factors of trait EI (emotionality, sociability, self-control, and wellbeing). The results of these regressions in the $\mathrm{TD}\left[\mathrm{F}(4,225)=74.37, \mathrm{p}<.001, \mathrm{R}^{2}=0.57\right]$ and autistic $\left[\mathrm{F}(4,273)=60.92, \mathrm{p}<.001, \mathrm{R}^{2}=0.47\right]$ samples can be seen in Supplementary Table 3. Emotionality was a significant predictor $(\beta=$ $-10.20, p<.001)$ in the TD sample, while emotionality $(\beta=-0.48, p<.001)$ and sociability $(\beta$ $=-0.20, p<.001)$ were significant predictors in the autistic sample.

\section{Personality, Trait EI and Camouflaging}

In the TD sample, there were negative correlations between camouflaging and agreeableness, extraversion, and trait EI. The relationship between camouflaging and extraversion was in the opposite direction to that proposed in Hypothesis 6. A positive correlation was found between neuroticism and camouflaging, in support of Hypothesis 7, and a negative correlation between conscientiousness and camouflaging, in support of Hypothesis 8. However, only the relationship between neuroticism and camouflaging was significant in the autistic sample, again supporting Hypothesis 7. Despite no overall association between camouflaging and extraversion in the autistic sample, a significant correlation was found with the camouflaging subscale of assimilation.

A multiple regression was undertaken of camouflaging in the TD sample, the results of which are presented in Table 4. This contained the subscales of BAPQ, the five-factor model of personality traits, and the factors of Trait EI as predictors. This model was significant $\left[F(12,217)=23.74, \mathrm{p}<.001 \mathrm{R}^{2}=.75\right]$ with pragmatic language, aloofness, rigidity, and self-control as significant predictors, in contrast to Hypotheses 6-8. 


\section{Table 4}

Multiple Regression Analyses Predicting Camouflaging from BAPQ subscales (Step 1), Big Five Personality (Step 2), and the Trait EI Factors (Step 3) in the Typically Developing Sample.

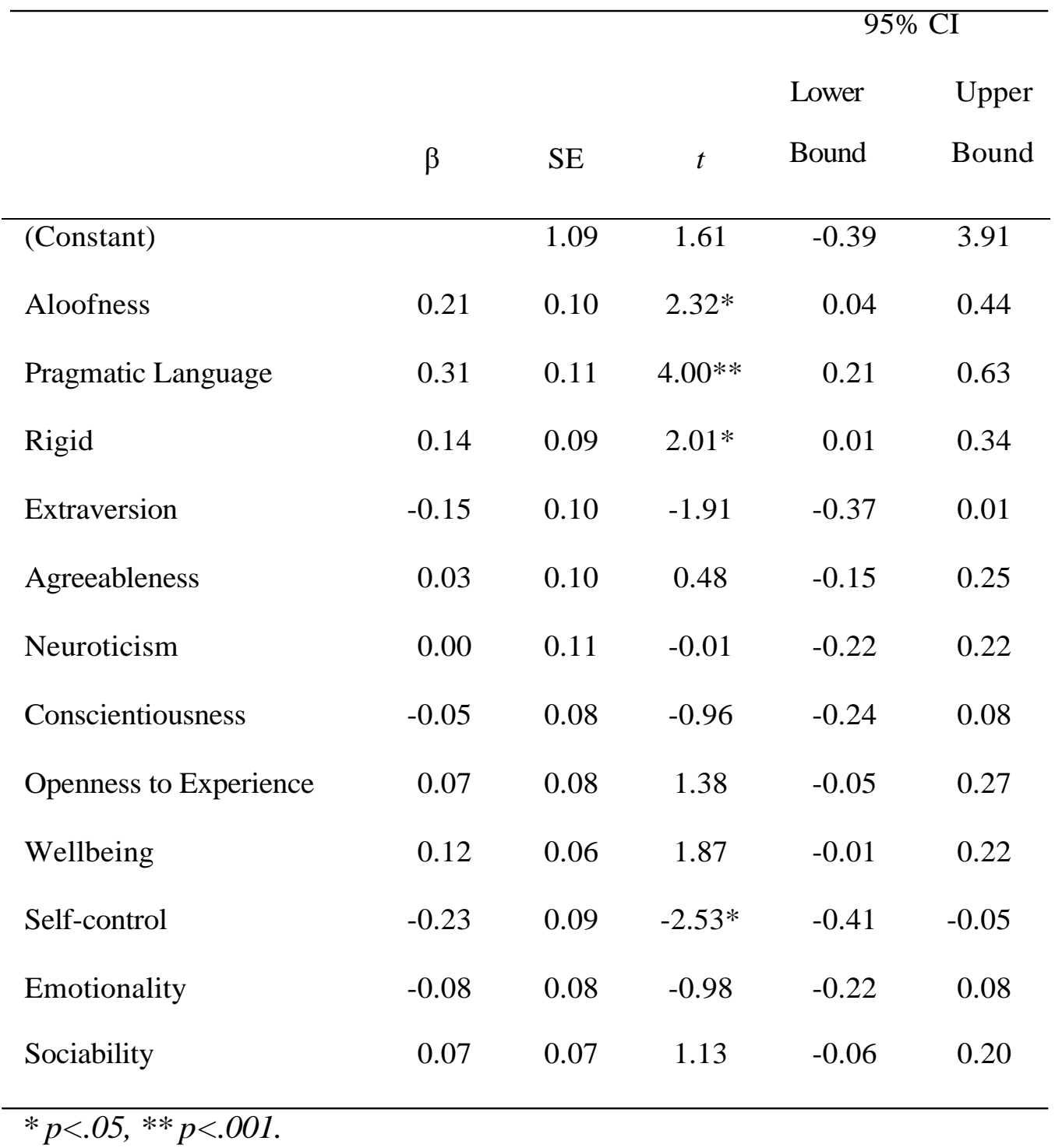

An identical multiple regression was undertaken in the autistic sample. This regression was significant $\left[F(12,264)=6.24, \mathrm{p}<.001, \mathrm{R}^{2}=.221\right]$ and the results can be seen in Table 5. Neuroticism was a predictor, in support of Hypothesis 7, in addition to aloofness, openness to experience, and wellbeing. 
Table 5

Multiple Regression Analyses Predicting Camouflaging from BAPQ subscales (Step 1), Big Five Personality (Step 2), and Trait EI subscales (Step 3) in the Autistic sample.

\begin{tabular}{|c|c|c|c|c|c|}
\hline \multirow[b]{3}{*}{ Predictor } & \multirow[b]{3}{*}{$\beta$} & \multirow[b]{3}{*}{ SE } & \multicolumn{3}{|c|}{$95 \% \mathrm{CI}$} \\
\hline & & & & Lower & Upper \\
\hline & & & $t$ & Bound & Bound \\
\hline (Constant) & & 1.15 & -0.13 & -2.42 & 2.12 \\
\hline Aloofness & 0.30 & 0.11 & $3.41 * *$ & 0.16 & 0.59 \\
\hline Pragmatic Language & -0.01 & 0.10 & -0.16 & -0.22 & 0.19 \\
\hline Rigid & 0.11 & 0.09 & 1.60 & -0.03 & 0.31 \\
\hline Extraversion & -0.04 & 0.10 & -0.50 & -0.24 & 0.14 \\
\hline Agreeableness & 0.06 & 0.09 & 0.92 & -0.09 & 0.25 \\
\hline Neuroticism & 0.23 & 0.12 & $2.58^{*}$ & 0.07 & 0.52 \\
\hline Conscientiousness & -0.08 & 0.08 & -1.38 & -0.27 & 0.05 \\
\hline Openness to Experience & 0.27 & 0.08 & $4.59 * *$ & 0.21 & 0.54 \\
\hline Wellbeing & 0.18 & 0.05 & $2.41^{*}$ & 0.02 & 0.24 \\
\hline Self-control & -0.07 & 0.08 & -0.82 & -0.23 & 0.10 \\
\hline Emotionality & 0.03 & 0.07 & 0.45 & -0.11 & 0.17 \\
\hline Sociability & 0.03 & 0.06 & 0.41 & -0.10 & 0.15 \\
\hline
\end{tabular}

\section{Discussion}

This study investigated the relationships between the five-factor model of personality, trait EI, autistic traits, and camouflaging.

$\underline{\text { Five-Factor Model and Autistic Traits }}$ 
A positive correlation between BAPQ scores and neuroticism was found across both groups. BAPQ scores were negatively correlated with extraversion and agreeableness across both groups. These results corroborate the findings of previous studies on both TD and autistic groups (Lodi-Smith et al., 2018) and mostly support the first hypothesis. As these associations have been consistently demonstrated, it suggests that they are particularly robust.

The correlations between conscientiousness and BAPQ scores were not significant in either the autistic or the TD sample. This contrasts with previous findings (Lodi-Smith et al. 2018), suggesting the relationship may require further examination. The relationship between autism and openness to experience has previously been shown to be a weak association (Lodi-Smith et al., 2018), which was supported in the current study.

These results suggest that there are specific personality dimensions underlying autistic traits. The findings of low extraversion, low agreeableness, and high neuroticism associated with high autistic traits were replicated across all analyses, suggesting these are particularly robust.

\section{$\underline{\text { Trait EI and Autistic Traits }}$}

A negative association between trait EI and autistic traits was hypothesized. Considerable support was found for this hypothesis with large correlations noted across both the autistic and the TD samples. This finding lends support to previous research in the field reporting a significantly lower level of trait EI in autistic samples (Boily et al., 2017; Gökçen et al., 2014). These findings have implications, especially for people high in autistic traits or diagnosed with ASD, as trait EI is an important factor for a fulfilling life (Petrides et al., 2016). Trait EI has also been related to treatment outcomes in depression and anxiety (Rudenstine \& Espinosa, 2018), suggesting that low trait EI may impact the recovery of autistic individuals suffering from these conditions. 
The regression results indicate that a large amount of variance in autistic traits can be accounted for by trait EI. This further supports the theory that difficulties with trait EI are a key characteristic of ASD. The analyses indicate that emotionality is a particularly good predictor of autistic traits, which is consistent with previous research (Gökçen et al., 2014). The high significance of the emotionality factor as a predictor of autistic traits in both TD and autistic samples suggests that an intervention specifically targeting emotionality would benefit individuals in both groups.

\section{Personality, Trait EI and Camouflaging}

Contrary to Hypothesis 3, there was a significant negative correlation between extraversion and camouflaging in the TD sample. It may be that introverted individuals feel they need to camouflage in social situations in order to appear more extraverted. However, this correlation was not significant in the autistic sample. This suggests camouflaging may not be the same as the self-presentation strategies used in TD populations, especially since the latter are positively related to with extraversion (Michikyan et al., 2014). Some personality traits may predict variation in self-presentation by TD individuals, whereas other factors, including situational variables, may underlie autistic individuals' camouflaging (Hull et al., 2018). However, extraversion did not significantly predict camouflaging beyond the role of autistic traits in either group, suggesting that the strong relationship between extraversion and autistic traits in the TD sample may account for some of this variance.

There was support for the hypothesis that neuroticism would have a positive association with camouflaging across both groups. Additionally, neuroticism and openness to experience both significantly predicted camouflaging beyond the influence of autistic traits in the autistic sample. This echoes previous findings that more neurotic individuals engage more often in self-presentation strategies (Michikyan et al., 2014; Seidman, 2013). In contrast to 
the results on extraversion, this shows a similarity between camouflaging and selfpresentation strategies. It may be that the two constructs are not completely synonymous, but that specific self-presentation strategies are related to camouflaging. It may also be that this association can be explained by the relationship between neuroticism and anxiety (Uliaszek et al., 2009), since previous research has shown that people who are more anxious are also more likely to engage in camouflaging (Hull et al., 2018).

Finally, it was predicted that individuals scoring higher on camouflaging would score lower on conscientiousness. In the TD sample, there was a significant negative correlation between total camouflaging and conscientiousness, as well as between assimilation and conscientiousness. This is in line with the results of Seidman (2013), where

conscientiousness was negatively associated with self-presentation behaviours. However, the correlation did not reach significance in the autistic group. These results again highlight a difference between the autistic group and the TD group in relation to personality traits associated with camouflaging. This may indicate a qualitative difference in camouflaging behaviours between individuals with and without a diagnosis of ASD, although again, the lack of a significant predictive effect of conscientiousness in the multiple regression means these findings should be replicated.

Although no specific hypotheses were advanced regarding the relationship between camouflaging and the personality domains of agreeableness and openness to experience, our results revealed a negative relationship with the former domain in the TD sample. However, this did not reach significance in the autistic group, further highlighting a pattern of difference between the TD group and autistic group. Openness to experience showed no significant correlations with camouflaging in any analysis, although it was a significant predictor of camouflaging in the autistic sample. Replication of these results is needed to 
better understand the relationship between camouflaging and this factor of personality in both autistic and TD groups.

Although not stated as a hypothesis, the relationship between trait EI and camouflaging was also explored. A negative correlation between the two variables was found in TD participants, which did not reach significance in the autistic sample. These findings suggest camouflaging is qualitatively different to self-presentation strategies, as trait EI has been shown to be positively related to self-presentation strategies (Petrides et al., 2007; Schutte et al., 2001). This may also indicate that more complex relationships exist between autism, camouflaging, and trait EI. When entered into a multiple regression with other correlates of camouflaging (BAPQ scores and BFI scores), wellbeing predicted camouflaging in autistic participants and self-control predicted camouflaging in TD participants. This suggests that trait EI may be partially predictive of camouflaging independent of the individual's level of autistic traits. However, more detailed empirical investigations need to be undertaken to understand the nature of this relationship and any other factors which may moderate or mediate it.

\section{$\underline{\text { Predicting Camouflaging }}$}

Camouflaging was regressed on BAPQ subscale scores, BFI variables, and the trait EI factors. In the TD sample, a model containing pragmatic language, aloofness, rigidity, and self-control accounted for the greatest variance in camouflaging. This suggests that camouflaging can be best predicted by autistic traits in TD individuals, and that other individual differences, including personality and trait EI, may impact it indirectly through their relationship with autistic traits. However, the significance of the self-control factor suggests that trait EI may influence camouflaging independent of autistic traits. In autistic participants, a model containing aloofness, neuroticism, openness to experience, and 
wellbeing accounted for the greatest variance in camouflaging. This suggests that camouflaging in autistic participants can be partially explained by some aspects of their personality and trait EI, in addition to their autistic traits. The common factor of aloofness in both models suggests that this aspect of the Broader Autism Phenotype may drive camouflaging in both TD and autistic individuals. Feeling separate to others may lead to use of camouflaging strategies in order to socialise and integrate (Hull et al., 2017).

\section{$\underline{\text { Strengths and Limitations }}$}

This study benefited from a large sample of autistic participants, which is rare in this area of research. As such, it offers some of the strongest evidence for the relationships between personality, trait EI, and autistic traits to date. Additionally, it is the first study to explore the role of personality and trait EI in camouflaging, with important implications for our understanding of the factors promoting camouflaging in both autistic and TD individuals.

A limitation of the current study is that, due to its online nature, participants' selfreported ASD diagnoses could not be verified, although participants were only included if they were able to report the type of healthcare professional who had diagnosed them. Autistic traits were used as a proxy of the types of autistic characteristics which may be camouflaged, but do not reflect scores on diagnostic assessments of autistic characteristics. However, scores on the BAPQ were significantly higher for the autistic group than the TD group, and scores in both groups were evaluated against the cut-off scores outlined in Sasson et al. (2013). Considerably more participants who self-reported as TD were below the cut-off threshold than those who self-reported as autistic.

\section{Conclusions}

This study aimed to investigate the relationship between autistic traits, the five-factor model of personality, trait EI, and camouflaging, in one of the largest samples yet in this area of 
research. Trait EI was negatively associated with autistic traits in both autistic and TD samples. This has implications for all individuals high in autistic traits, as trait EI is strongly linked to psychological wellbeing and life satisfaction. Differences in the predictors of camouflaging were found between autistic and TD samples, suggesting that autistic camouflaging may be partially driven by situational pressures, such as stigma, and TD selfpresentation strategies may be driven by individual differences, especially autistic traits. 


\section{Funding}

This research did not receive any specific grant from funding agencies in the public, commercial, or not-for-profit sectors. 


\section{References}

American Psychiatric Association. (2013). Diagnostic and statistical manual of mental disorders (5th ed.). Arlington, VA: American Psychiatric Publishing.

Baron-Cohen, S., \& Wheelwright, S. (2004). The empathy quotient: an investigation of adults with Asperger syndrome or high functioning autism, and normal sex differences. Journal of Autism and Developmental Disorders, 34(2), 163-175.

Boily, R., Kingston, S. E., \& Montgomery, J. M. (2017). Trait and ability emotional intelligence in adolescents with and without autism spectrum disorder. Canadian Journal of School Psychology, 32(3-4), 282-298.

Gökçen, E., Petrides, K. V., Hudry, K., Frederickson, N., \& Smillie, L. D. (2014). Sub threshold autism traits: The role of trait emotional intelligence and cognitive flexibility. British Journal of Psychology, 105(2), 187-199.

Golan, O., Baron-Cohen, S., \& Hill, J. (2006). The Cambridge mindreading (CAM) facevoice battery: Testing complex emotion recognition in adults with and without Asperger syndrome. Journal of Autism and Developmental Disorders, 36(2), 169-183.

Hull, L., Petrides, K. V., Allison, C., Smith, P., Baron-Cohen, S., Lai, M. C., \& Mandy, W. (2017). "Putting on My Best Normal": Social Camouflaging in Adults with Autism Spectrum Conditions. Journal of Autism and Developmental Disorders, 47(8), 2519- 
Hull, L., Mandy, W., Lai, M.C., Baron-Cohen, S., Allison, C., Smith, P. \& Petrides, K. V. (2018). Development and validation of the Camouflaging Autistic Traits Questionnaire (CAT-Q). Journal of Autism and Developmental Disorders, 1-15.

Hurley, R. S., Losh, M., Parlier, M., Reznick, J. S., \& Piven, J. (2007). The broad autism phenotype questionnaire. Journal of Autism and Developmental Disorders, 37(9), 1679-1690.

Izuma, K., Matsumoto, K., Camerer, C. F., \& Adolphs, R. (2011). Insensitivity to social reputation in autism. Proceedings of the National Academy of Sciences, 108(42), 17302-17307.

John, O. P., \& Srivastava, S. (1999). The Big-Five trait taxonomy: History, measurement, and theoretical perspectives. In L. A. Pervin \& O. P. John (Eds.), Handbook of personality: Theory and research (Vol. 2, pp. 102138). New York: Guilford Press.

John, O. P., Cheek, J. M., \& Klohnen, E. C. (1996). On the nature of self-monitoring: Construct explication with Q-sort ratings. Journal of Personality and Social Psychology, 71, 763-776.

Kenny, L., Hattersley, C., Molins, B., Buckley, C., Povey, C., \& Pellicano, E. (2016). Which terms should be used to describe autism? Perspectives from the UK autism community. Autism, 20(4), 442-462.

Leary, M. R., \& Kowalski, R. M. (1990). Impression management: A literature review and two-component model. Psychological Bulletin1, 107(1), 3447. 
Lodi-Smith, J., Rodgers, J. D., Cunningham, S. A., Lopata, C., \& Thomeer, M. L. (2018). Meta-analysis of Big Five personality traits in autism spectrum disorder. Autism, 1362361318766571.

Michikyan, M., Subrahmanyam, K., \& Dennis, J. (2014). Can you tell who I am? Neuroticism, Extraversion, and online self-presentation among young adults. Computers in Human Behavior, 33, 179-183.

Petrides, K. V. (2009). Psychometric properties of the Trait Emotional Intelligence Questionnaire. In C. Stough, D. H. Saklofske, and J. D. Parker, Advances in the assessment of emotional intelligence. New York: Springer. DOI: 10.1007/978-0-38788370-0_5

Petrides, K. V., Hudry, K., Michalaria, G., Swami, V., \& Sevdalis, N. (2011). A comparison of the trait emotional intelligence profiles of individuals with and without Asperger syndrome. Autism, 15(6), 671-682.

Petrides, K. V., Mikolajczak, M., Mavroveli, S., Sanchez-Ruiz, M. J., Furnham, A., \& PérezGonzález, J. C. (2016). Developments in trait emotional intelligence research. Emotion Review, 8(4), 335-341.

Petrides, K. V., Pérez-González, J. C., \& Furnham, A. (2007). On the criterion and incremental validity of trait emotional intelligence. Cognition and Emotion, 21(1), 26-

55. 
in personality factor space. British Journal of Psychology, 98(2), 273-289.

Rotatori, A. F., \& Deisinger, J. A. (Eds.). (2015). The Broad Autism Phenotype. Emerald Group Publishing.

Rudenstine, S., \& Espinosa, A. (2018). Examining the role of trait emotional intelligence on psychiatric symptom clusters in the context of lifetime trauma. Personality and Individual Differences, 128, 69-74.

Sasson, N. J., Lam, K. S., Childress, D., Parlier, M., Daniels, J. L., \& Piven, J. (2013). The Broad Autism Phenotype Questionnaire: Prevalence and Diagnostic Classification. Autism Research, 6(2), 134-143.

Schutte, N. S., Malouff, J. M., Bobik, C., Coston, T. D., Greeson, C., Jedlicka, C., Rhodes, E., \& Wendorf, G. (2001). Emotional intelligence and interpersonal relations. The Journal of Social Psychology, 141(4), 523-536.

Seidman, G. (2013). Self-presentation and belonging on Facebook: How personality influences social media use and motivations. Personality and Individual Differences, 54(3), 402-407.

Soto, C. J., \& John, O. P. (2009). Ten facet scales for the Big Five Inventory: Convergence with NEO PI-R facets, self-peer agreement, and discriminant validity. Journal of Research in Personality, 43(1), 84-90.

Trapnell, P. D., \& Campbell, J. D. (1999). Private self-consciousness and the Five-factor model of personality: distinguishing rumination from reflection. Journal of 
Personality and Social Psychology, 76(2), 284.

Uliaszek, A. A., Hauner, K. K., Zinbarg, R. E., Craske, M. G., Mineka, S., Griffith, J. W., \& Rose, R. D. (2009). An examination of content overlap and disorder-specific predictions in the associations of neuroticism with anxiety and depression. Journal of Research in Personality, 43(5), 785-794. 
Supplementary Material

Click here to download Supplementary Material: Supplementary Material.docx

Table 2

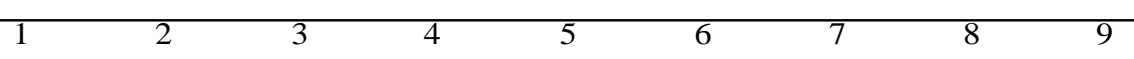




\begin{tabular}{|c|c|c|c|c|c|c|c|c|c|c|c|c|c|c|c|c|c|c|}
\hline 1. BAPQ & - & - & - & - & $-.438 *$ &.$- .381 *$ & $.378 *$ & -.006 & -.193 & $.310 *$ & .190 & -.022 & $.651^{*}$ & $-.654 *$ & $-.381 *$ & $-.429 *$ & $-.622 *$ & $-.463 *$ \\
\hline 2. Aloofness & - & - & $.532 *$ & $.517 *$ & $-.569 *$ & $-.358 *$ & $.252 *$ & .034 & -.165 & $.303 *$ & .111 & .034 & $.688 *$ & $-.569 *$ & $-.386 *$ & $-.283 *$ & $-.575^{*}$ & $-.429 *$ \\
\hline 3. Pragmatic Language & - & $.663 *$ & - & $.503 *$ & $-.254 *$ &.$- .307 *$ & $.327 *$ & -.130 & -.108 & .218 & .166 & -.062 & $.462 *$ & $-.571 *$ & $-.286 *$ & $-.411 *$ & $-.546 *$ & $-.437 *$ \\
\hline 4. Rigid & - & $.587 *$ & $.615^{*}$ & - & $-.249 *$ & $-.272 *$ & $.354 *$ & .065 & -.198 & $.242 *$ & .192 & -.03 & $.454 *$ & $-.482 *$ & $-.269 *$ & $-.372 *$ & $-.421 *$ & $-.286 *$ \\
\hline 5. Extraversion & $-.600 *$ & $-.719 *$ & $-.426 *$ & $-.383 *$ & - & .072 & $-.309 *$ & .069 & $.251 *$ & -.170 & .017 & -.026 & $-.503 *$ & $.485 *$ & $.337 *$ & $.251 *$ & $.351 *$ & $.456 *$ \\
\hline 6. Agreeableness & $-.563 *$ & $-.545 *$ & $-.444 *$ & $-.463 *$ & $.300 *$ & - & $-.266 *$ & .114 & $.227 *$ & -.018 & -.003 & .092 & -.155 & $.374 *$ & $.394 *$ & $.231 *$ & $.339 *$ & .108 \\
\hline 7. Neuroticism & $.503 *$ & $.367 *$ & $.441 *$ & $.507 *$ & $-.379 *$ & $-.440 *$ & - & -.148 & -.199 & $.229 *$ & .111 & .084 & $.418 *$ & $-.641 *$ & $-.552 *$ & $-.731 *$ & $-.258 *$ & $-.419 *$ \\
\hline 8. Conscientiousness & -.204 & -.139 & -.313 & -.091 & .101 & $.271 *$ & $-.362 *$ & - & .164 & -.033 & .027 & -.083 & -.048 & $.284 *$ & .175 & $.302 *$ & .111 & .113 \\
\hline 9. Openness & -.164 & -.097 & -.072 & $-.256 *$ & .224 & .119 & -.234 & .003 & - & .186 & $.261 *$ & .150 & -.026 & $.313 *$ & $.243 *$ & .217 & .223 & .168 \\
\hline 10. Camouflaging & $.714 *$ & $.628 *$ & $.676 *$ & $.552 *$ & $-.471 *$ & $-.399 *$ & $.439 *$ & $-.252 *$ & -.058 & - & - & - & - & -.179 & -.046 & -.220 & -.138 & -.150 \\
\hline 11. Compensation & $.645 *$ & $.509 *$ & $.664 *$ & $.512 *$ &.$- .351 *$ & $-.352 *$ & $.322 *$ & -.205 & .007 & - & - & $.613 *$ & $.401 *$ & -.028 & .104 & -.109 & -.072 & -.021 \\
\hline 12. Masking & $.271 *$ & .227 & .231 & $.246^{*}$ & -.142 & -.128 & .234 & -.111 & -.045 & - & $.562 *$ & - & $.334 *$ & .057 & .035 & -.067 & .143 & .041 \\
\hline 13. Assimilation & $.817 *$ & $.800 *$ & $.728 *$ & $.583 *$ & $-.664 *$ & $-.487 *$ & $.544 *$ &.$- .304 *$ & -.124 & - & $.671 *$ & $.443 *$ & - & $-.552 *$ & $-.335 *$ & $-.409 *$ & $-.461 *$ & $-.459 *$ \\
\hline 14. Trait EI & $-.727 *$ & $-.628 *$ & $-.691 *$ & $-.574 *$ & $.605 *$ & $.515 *$ & $-.712 *$ & $.361 *$ & $.289 *$ & $-.588 *$ & $-.495 *$ & -.195 & $-.739 *$ & - & - & - & - & - \\
\hline 15. Wellbeing & $-.481 *$ & $-.445 *$ & $-.422 *$ &.$- .379 *$ & $.506 *$ & $.389 *$ & $-.597 *$ & $.301 *$ & .241 & $-.350 *$ & $-.268 *$ & -.056 & $-.520 *$ & - & - & $.458 *$ & $.398 *$ & $.417 *$ \\
\hline 16. Self-control & $-.510 *$ &.$- .358 *$ & $-.515 *$ & $-.464 *$ & $.315 *$ & $.383 *$ & $-.823 *$ & $.438 *$ & $.252 *$ & $-.497 *$ &.$- .393 *$ & $-.284 *$ & $-.566 *$ & - & $.574 *$ & - & $.350 *$ & $.430 *$ \\
\hline 17. Emotionality & $-.720 *$ & $-.672 *$ & $-.680 *$ & $-.516 *$ & $.524 *$ & $.545 *$ & $-.392 *$ & .084 & .151 & $-.564 *$ & $-.522 *$ & -.168 & $-.667 *$ & - & $.487 *$ & $.473^{*}$ & - & $.374 *$ \\
\hline 18. Sociability & $-.525 *$ & $-.493 *$ & $-.498 *$ & $-.372 *$ & $.580 *$ & $.260 *$ & $-.496 *$ & $.246^{*}$ & $.280 *$ & $-.407 *$ & $-.332 *$ & -.095 & $-.555 *$ & - & $.520 *$ & $.535^{*}$ & $.527 *$ & - \\
\hline
\end{tabular}

Summary of Intercorrelations for the TD Sample (below diagonal) and the Autistic Sample (above diagonal)

* adjusted p<.05. BAPQ = Broad Autism Phenotype Questionnaire (total score); Trait EI = Trait Emotional Intelligence (total score) 
Table 3

Regression of BAPQ Scores on Trait EI Factors in TD and Autistic Samples

\begin{tabular}{|c|c|c|c|c|}
\hline $\begin{array}{l}\text { TD Sample } \\
(\mathrm{N}=230)\end{array}$ & $\beta$ & $\mathrm{SE}$ & $p$ & $95 \% \mathrm{CI}$ \\
\hline (Constant) & 5.91 & 0.17 & $<.001$ & $5.57-6.25$ \\
\hline Wellbeing & -0.07 & 0.04 & 0.262 & $-0.11-0.03$ \\
\hline Self-control & -2.50 & 0.04 & 0.013 & $-0.18--0.02$ \\
\hline Emotionality & -10.20 & 0.04 & $<.001$ & $-0.45--0.30$ \\
\hline Sociability & -2.12 & 0.04 & 0.035 & $-0.16--0.01$ \\
\hline \multicolumn{5}{|c|}{$F(4,225)=74.37, \mathrm{p}<.001, \mathrm{R}^{2}=0.57$} \\
\hline $\begin{array}{l}\text { Autistic Sample } \\
\qquad(\mathrm{N}=278)\end{array}$ & $\beta$ & SE & $p$ & $95 \% \mathrm{CI}$ \\
\hline (Constant) & 6.09 & 0.12 & $<.001$ & $5.85-6.33$ \\
\hline Wellbeing & -0.03 & 0.03 & 0.53 & $-0.07-0.03$ \\
\hline Self-control & -0.16 & 0.03 & 0.002 & $-0.16--0.04$ \\
\hline Emotionality & -0.48 & $\mathbf{0 . 0 3}$ & $<.001$ & $-0.36--0.23$ \\
\hline Sociability & -0.20 & 0.03 & $<.001$ & $-0.17--0.06$ \\
\hline
\end{tabular}

Note. $\mathrm{CI}=$ confidence interval. Tests were assessed against $p=0.00227$ to correct for multiple comparisons. 


\section{Author Contributions}

Erin Robinson: Conceptualisation, Methodology, Investigation, Formal Analysis, Writing - Original Draft. Laura Hull: Resources, Writing - Review \& Editing, Supervision. KV Petrides: Conceptualisation, Writing - Review \& Editing, Supervision. 